OPEN ACCESS

Edited by:

John Doonan,

Aberystwyth University, UK

Reviewed by:

Hao Peng,

Washington State University, USA

Konstantinos Vlachonasios,

Aristotle University of Thessaloniki,

Greece

${ }^{*}$ Correspondence:

Klára Panzarová

panzarova@psi.cz

Magdalena M. Julkowska

magdalena.julkowska@kaust.edu.sa

Specialty section:

This article was submitted to

Crop Science and Horticulture,

a section of the journal

Frontiers in Plant Science

Received: 30 April 2016

Accepted: 05 September 2016

Published: 28 September 2016

Citation:

Awlia M, Nigro A, Fajkus J,

Schmoeckel SM, Negrão S,

Santelia D, Trtilek M, Tester M, Julkowska MM and Panzarová K

(2016) High-Throughput

Non-destructive Phenotyping of Traits that Contribute to Salinity Tolerance

in Arabidopsis thaliana.

Front. Plant Sci. 7:1414.

doi: 10.3389/fpls.2016.01414

\section{High-Throughput Non-destructive Phenotyping of Traits that Contribute to Salinity Tolerance in Arabidopsis thaliana}

\author{
Mariam Awlia', Arianna Nigro², Jiři Fajkus ${ }^{3}$, Sandra M. Schmoeckel', Sónia Negrão ${ }^{1}$, \\ Diana Santelia ${ }^{2}$, Martin Trtílek$^{3}$, Mark Tester ${ }^{1}$, Magdalena M. Julkowska $^{1 *}$ and \\ Klára Panzarová ${ }^{*}$ \\ 'Division of Biological and Environmental Sciences and Engineering, King Abdullah University of Science and Technology, \\ Thuwal, Saudi Arabia, ${ }^{2}$ Institute of Plant and Microbial Biology, University of Zurich, Zurich, Switzerland, ${ }^{3}$ PSI (Photon \\ Systems Instruments), Drásov, Czech Republic
}

Reproducible and efficient high-throughput phenotyping approaches, combined with advances in genome sequencing, are facilitating the discovery of genes affecting plant performance. Salinity tolerance is a desirable trait that can be achieved through breeding, where most have aimed at selecting for plants that perform effective ion exclusion from the shoots. To determine overall plant performance under salt stress, it is helpful to investigate several plant traits collectively in one experimental setup. Hence, we developed a quantitative phenotyping protocol using a high-throughput phenotyping system, with RGB and chlorophyll fluorescence (ChIF) imaging, which captures the growth, morphology, color and photosynthetic performance of Arabidopsis thaliana plants in response to salt stress. We optimized our salt treatment by controlling the soil-water content prior to introducing salt stress. We investigated these traits over time in two accessions in soil at 150,100 , or $50 \mathrm{mM} \mathrm{NaCl}$ to find that the plants subjected to $100 \mathrm{mM} \mathrm{NaCl}$ showed the most prominent responses in the absence of symptoms of severe stress. In these plants, salt stress induced significant changes in rosette area and morphology, but less prominent changes in rosette coloring and photosystem II efficiency. Clustering of ChIF traits with plant growth of nine accessions maintained at $100 \mathrm{mM} \mathrm{NaCl}$ revealed that in the early stage of salt stress, salinity tolerance correlated with non-photochemical quenching processes and during the later stage, plant performance correlated with quantum yield. This integrative approach allows the simultaneous analysis of several phenotypic traits. In combination with various genetic resources, the phenotyping protocol described here is expected to increase our understanding of plant performance and stress responses, ultimately identifying genes that improve plant performance in salt stress conditions.

Keywords: high-throughput phenotyping, Arabidopsis thaliana, salt stress, salinity tolerance, shoot-ion independent tolerance, kinetic chlorophyll fluorescence imaging, color segmentation 


\section{INTRODUCTION}

Climate change and population growth place a twofold pressure on agricultural crop production. Crop yields need to be sustained and increased while grown in unfavorable environments (Godfray et al., 2010; Tester and Langridge, 2010; Tilman et al., 2011). To meet future food demands, breeding efforts are targeting more resource-efficient and stresstolerant crops by combining large-scale plant phenotyping with genome sequencing in forward genetics studies. Phenotypic traits, including growth rate, size, shape, color, temperature and photosynthetic activity, are traditionally studied to evaluate plant performance under stress (Sirault et al., 2009; Zhang et al., 2012; Hairmansis et al., 2014; Chen et al., 2015; Ghanem et al., 2015). Plant breeding programs aimed at enhancing plant performance should investigate growth and photosynthetic activity in tandem because these processes are interdependent (Longenberger et al., 2009).

Advances in non-destructive image-based phenotyping technologies are enabling parallel studies of plant growth and photosynthetic performance over time (Munns et al., 2010; Dhondt et al., 2013; Hairmansis et al., 2014) using RGB and chlorophyll fluorescence (ChlF) imaging (Dhondt et al., 2013; Brown et al., 2014; Humplik et al., 2015). Traits related to plant growth, architecture and development have been quantified from digital color imaging, while leaf color is a simple, under-utilized trait that indicates plant health and leaf senescence (Berger et al., 2012). Kinetic ChlF imaging is a powerful tool for measuring plant photosynthetic capacity and provides valuable insights into the performance of photosynthetic apparatus (Oxborough, 2004; Baker, 2008). Light energy, captured by chlorophyll molecules, can undergo one of three fates: (1) be used to drive photosynthesis by photochemistry, (2) be dissipated as heat, or (3) be re-emitted as fluorescence. Because these three processes co-exist in close competition, the ChlF yield provides information on both the quantum efficiency of the plant's photochemistry and on the amount of heat dissipated. Under conditions of stress, the photochemical yield decreases, which in turn, causes heat dissipation and ChlF emissions to increase (Maxwell and Johnson, 2000; Murchie and Lawson, 2013). Although, high-throughput phenotyping of photosynthetic performance has previously been employed to study plant response to cold (Jansen et al., 2009; Humplik et al., 2015) and drought (Bresson et al., 2015), few studies have established an integrative approach that simultaneously analyzes plant growth and photosystem II (PSII) efficiency (Humplik et al., 2015). Systems for kinetic ChlF imaging have not been widely integrated into high-throughput phenotyping platforms, in contrast to single-level steady-state ChlF imaging. The latter only reflects chlorophyll content and not PSII activity. This means that using single-level steady-state ChlF can only discriminate between healthy, senescing and stressed leaves by the amount of chlorophyll they degrade (Campbell et al., 2015).

Soil salinity is a key stress factor that affects agriculture on a global scale (Munns and Tester, 2008; Cabot et al., 2014; Roy et al., 2014). In saline soil, plants accumulate ions in their shoots, compromising plant growth with ion toxicity, for example by reducing the rate of photosynthesis (Munns and Tester, 2008). During the early phase of salt stress, before ions accumulate significantly in the shoots, the osmotic phase of salinity tolerance takes place, which is referred to as shoot ion-independent tolerance (SIIT; Roy et al., 2014). During this phase, growth reduction is caused by decreased leaf emergence and expansion (Fricke et al., 2006; Munns and Tester, 2008; Berger et al., 2012). Mechanisms underlying ion sensing, cell cycle, cell expansion, and stomatal conductance are likely underlying this response (Ma et al., 2006; Stephan and Schroeder, 2014). Thus, performing regular growth measurements from when salt stress is introduced to the plant across an extended period of time provides the opportunity to discriminate between early (osmotic) and late (ionic) growth-related responses to salt stress. During the early phase of salt stress, the capacity of photosynthetic machinery is reduced (James et al., 2006; Chaves et al., 2009). In later phases, excessive photonic energy causes photochemical inactivation (Muranaka et al., 2002) that reduces PSII stability (Stepien and Johnson, 2009), limits stomatal gas diffusion and causes changes in carbon assimilation rates (Chaves et al., 2009), ultimately resulting in decreased photosynthetic activity. Additional limits to photosynthesis may be caused by the accumulation of unused organic compounds from carbon assimilation (Munns and Tester, 2008). Early responses of plants to salinity have previously been quantified by measuring rosette area, color, temperature, and photosynthetic activity using steady-state ChlF (Rajendran et al., 2009; Sirault et al., 2009; Berger et al., 2012; Chen et al., 2015). Here, we quantify how traits related to plant morphology, color and photosynthetic activity respond to salinity in one experimental setup to establish significant correlations among individual phenotypes.

We developed a phenotyping method that monitors plant responses to salt stress by evaluating plant growth, color and photosynthetic traits using an automated, high-throughput system. We grew Arabidopsis plants in soil and maintained them at 40,60 , or $80 \%$ of the soil-water holding capacity to achieve approximately 150,100, and $50 \mathrm{mM} \mathrm{NaCl}$, respectively. We established $100 \mathrm{mM} \mathrm{NaCl}$ as the optimal condition for salt treatment. To characterize the early and late plant responses to salt stress, we investigated RGB, greenness and photosynthesisrelated traits. Traits of $\mathrm{ChlF}$ were clustered with relative plant performance values into groups corresponding to early and late responses to salt stress. This work provides the means for screening natural diversity panels and mapping populations to identify candidate genes underlying plant development and stress tolerance.

\section{MATERIALS AND METHODS}

\section{Plant Material and Growing Conditions}

Accessions of Arabidopsis Columbia-0 (Col-0) and C24 were used to establish the cultivation, salt treatment and phenotyping protocol. Thereafter, nine accessions of Arabidopsis [Col0, C24, Canary Islands (Can), Coimbra (Co), Cape Verde Islands (Cvi), Landsberg erecta (Ler), Niederzenz (Nd), Rschew (Rsch) and Tenela (Te)] were used to optimize this protocol 
and investigate the natural variation of plants in response to salt stress (Hannah et al., 2006). Seeds were sown into pots $(70 \mathrm{~mm} \times 70 \mathrm{~mm} \times 65 \mathrm{~mm}$, Poppelman TEKU DE) containing $60 \mathrm{~g}$ of freshly sieved soil (Substrate 2, KlasmannDeilmann GmbH, Germany) and watered to full soil-water holding capacity. Seeds were stratified for 3 days at $4^{\circ} \mathrm{C}$ in the dark. All plants were grown in a climate controlled growth chamber (FS_WI, PSI, Czech Republic) with cool-white LED and far-red LED lighting. The protocol was setup with Col-0 and C24 grown in a $10 \mathrm{~h} / 14 \mathrm{~h} 21^{\circ} \mathrm{C} / 15^{\circ} \mathrm{C}$ light/dark cycle at a relative humidity of $60 \%$ and a photon irradiance of $250 \mu \mathrm{mol} \mathrm{m}^{-2} \mathrm{~s}^{-1}$. The protocol was optimized for the nine accessions using a $12 \mathrm{~h} / 12 \mathrm{~h} 22^{\circ} \mathrm{C} / 20^{\circ} \mathrm{C}$ light/dark cycle with a relative humidity of $55 \%$ and an irradiance of $150 \mu \mathrm{mol} \mathrm{m}{ }^{-2} \mathrm{~s}^{-1}$. Seven days after stratification (DAS), seedlings of similar size were transplanted into soil that had been watered 1 day in advance to full soil-water holding capacity. Plants were cultivated in the growth chamber until most plants were at the 10-leaf stage (24 DAS for plants in the $10 \mathrm{~h} / 14 \mathrm{~h}$ light/dark cycle and 21 DAS in the $12 \mathrm{~h} / 12 \mathrm{~h}$ light/dark cycle). The growth timeline for Col-0 and C24 plants illustrates the implementation of the three watering regimes, the salt treatment and the phenotyping protocol (Figure 1).

\section{Watering and Salt Treatment}

Similar to Junker et al. (2015), we determined the soil waterholding capacity by filling 10 pots with $60 \mathrm{~g}$ of sieved soil and drying them for 3 days at $80^{\circ} \mathrm{C}$ to completely desiccate the soil. Soil was then saturated with water and left to drain for 1 day before weighing. Based on the soil-water content at 100\% (130 g),
40,60 , and $80 \%$ of the soil-water holding capacity were found to weigh 52, 77, and 103 g, respectively. At 14 DAS, Col-0 and C24 seedlings were placed randomly in trays $(5 \times 4$ pots per tray) and into the PlantScreen ${ }^{\mathrm{TM}}$ Compact System (PSI, Czech Republic) and automatically weighed and watered every other day to reach and maintain the reference weight corresponding to the desired soil-water contents (Figure 1A). Once at the 10-leaf stage at 24 DAS, nine replicates per accession were placed in $250 \mathrm{mM} \mathrm{NaCl}$ or $\mathrm{dH}_{2} \mathrm{O}$ for $1 \mathrm{~h}$ to ensure full saturation of the soil. Pots were left to drain for $10 \mathrm{~min}$ before being placed in the phenotyping system. Effective $\mathrm{NaCl}$ concentration in the soil was estimated as 150,100 , and $50 \mathrm{mM} \mathrm{NaCl}$ in plants watered to 40,60 , and $80 \%$ soil-water holding capacity, respectively, representing conditions of severe, moderate, and mild salt stress (Figure 1B). For 11 days, with the exception of days 5 and 6 (Figure 1C), plants were transferred manually from the growth chamber to PlantScreen ${ }^{\mathrm{TM}}$ for image acquisition and then returned to the same positions inside the growth chamber. On the final day of imaging, the water content of the soil was found to be approximately 60$70 \%$, indicating that plants had adequate soil moisture during the phenotyping period.

After analyzing the effects of the three watering regimes, $60 \%$ soil-water content and $100 \mathrm{mM} \mathrm{NaCl}$ were established as effective conditions for investigating early responses to salt stress. We then optimized the protocol, in terms of growth conditions and ChlF imaging, using nine accessions of Arabidopsis. At 18 and 20 DAS pots were watered to the target soil-water content. Once at the 10-leaf stage, at 21 DAS, eight replicates per accession were placed in a $250 \mathrm{mM} \mathrm{NaCl}$ solution or $\mathrm{dH}_{2} \mathrm{O}$ for $1 \mathrm{~h}$ to
A

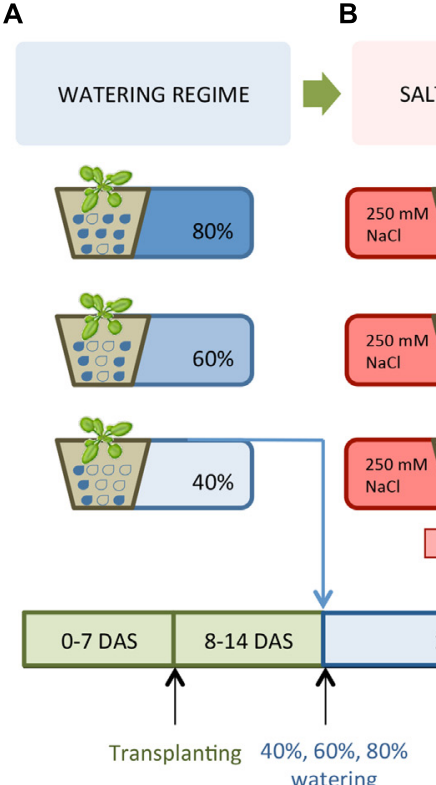

watering c
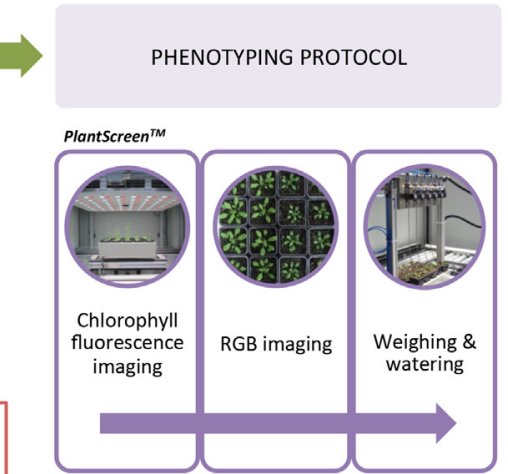

\begin{tabular}{llllllllllll}
1 & 2 & 3 & 4 & 5 & 6 & 7 & 8 & 9 & 10 & 11 \\
\hline & & & & & & & & & & &
\end{tabular}

24-28 DAS

31-34 DAS

FIGURE 1 | Watering regime, salt stress treatment and phenotyping protocol. (A) Col-0 and C24 were sown, watered to full soil-water saturation, stratified, then germinated under short day conditions. Similar-sized seedlings were transplanted into freshly sieved soil 7 days after stratification (DAS). At 14 DAS, watering was controlled to reach 80,60 , or $40 \%$ of the soil-water holding capacity. (B) Seedlings at the 10-leaf stage (24 DAS) were saturated for $1 \mathrm{~h}$ in a $250 \mathrm{mM} \mathrm{NaCl}$ solution to reach concentrations of 50,100 , and $150 \mathrm{mM} \mathrm{NaCl}$, while control pots were saturated in $\mathrm{dH}_{2} \mathrm{O}$. (C) The PlantScreen ${ }^{\mathrm{TM}}$ Compact System performed chlorophyll fluorescence (ChIF) and RGB imaging, as well as automatically weighing and watering the plants. The lower panel depicts the timeline of the experiment. 
ensure saturation of the soil. Plants were imaged for 7 days with no additional watering. The final soil-water content was approximately $70-80 \%$.

\section{High-Throughput Phenotyping}

Control and salt-treated plants were automatically phenotyped for RGB and kinetic ChlF traits using PlantScreen ${ }^{\mathrm{TM}}$ (Supplementary Figures S1 and S2) from $1 \mathrm{~h}$ after introducing salt stress. The phenotyping was conducted for 11 days to develop the protocol and 7 days to investigate natural variation among the nine accessions. Trays were transported within PlantScreen ${ }^{\mathrm{TM}}$ on conveyor belts between the light-isolated imaging cabinets, weighing and watering station and the dark/light acclimation chamber. A single round of measuring consisted of an initial 15 min dark-adaptation period inside the acclimation chamber, followed by ChlF and RGB imaging, weighing and watering. Pixel count, color and fluorescence intensity were evaluated from the images. A total time of $1 \mathrm{~h}$ and $40 \mathrm{~min}$ was required to measure 10 trays (200 plants). The PlantScreen ${ }^{\mathrm{TM}}$ Analyzer software (PSI, Czech Republic) was used to automatically process the raw data.

\section{RGB Imaging and Processing}

Trays were loaded into the imaging cabinet of the PlantScreen ${ }^{\text {TM }}$ platform with three RGB cameras (one top and two side views) mounted on robotic arms, each supplemented with an LED-based light source to ensure homogenous illumination of the imaged object. To assess plant growth and morphological traits, RGB images (resolution $2560 \times 1920$ pixels) of $5 \times 4$ plants per tray were captured using the GigE uEye UI-5580SEC/M 5 Mpx Camera (IDS, Germany) from the top view only. Light conditions, plant position and camera settings were fixed throughout the experiments. The PlantScreen ${ }^{\mathrm{TM}}$ software required three steps to extract features from the RGB images. (1) Basic processing applied in real-time involving correction for barrel (fisheye) distortion, tray detection, cropping of individual pots, background subtraction to remove nonplant pixels from the images, filtration and artifact removal to produce binary (black and white) and RGB representations of each plant [binary images represent the plant's surface (white) and background (black)]; (2) morphologic analysis, requiring separation of the background from the plant shoot tissue allowing the pixel number per plant and rosette area to be counted; and (3) analysis of greenness using background-subtracted RGB images to evaluate the color. For this step, the images were color-segmented to represent and evaluate rosette coloring (Supplementary Figure S1). The morphometric parameters area, perimeter, roundness, compactness, rotational mass symmetry, eccentricity and slenderness of leaves were computed from the RGB image processing and have been listed and defined in Supplementary Table S1.

\section{Plant Growth-Related Parameters}

To evaluate the effect of salt stress on early and late plant growth rates (GR), we examined the increase in projected rosette area over time by fitting a linear function to two time intervals. The regression coefficient of the fitted function was determined and used as a trait in the statistical analysis. Relative effects of salt stress were calculated by dividing the estimated growth rates (GR) in salt conditions by the average in control conditions

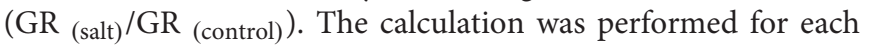
accession over two time intervals (0-4 days and then 7-11 days when developing the protocol, and 0-3 days and then 4-7 days when examining natural variation among accessions; Figures 2D and $5 \mathrm{C}$ ). This ratio has been termed the shoot ion-independent tolerance (SIIT) index, which is used as a measure of plant salinity tolerance (Roy et al., 2014). The effects of salt stress were determined by performing an analysis of variance (ANOVA) per accession and treatment with Tukey's post hoc test of significance for each RGB trait ( $p$-value $<0.05)$.

\section{Color Segmentation and Evaluation of Greenness}

Using color segmentation, we analyzed the change in rosette coloring. We calibrated the analysis by using RGB images from both control and salt-stressed conditions and from the start, middle and end of the phenotyping period to obtain an unbiased color scale (Supplementary Figure S1). Values in the RGB channels, from each pixel corresponding to the plant's surface area, were extracted to serve as a dataset for k-means clustering. Nine clusters were sufficient for optimal color differentiation and all input pixels were partitioned according to their Euclidean distance in the RGB color space. The RGB coordinates of cluster centroids were used as base hues to evaluate greenness. Original pixel color was approximated from the nearest cluster centroid, yielding color-segmented images. To calculate the relative hue abundance independent of the rosette area, pixel counts of individual hue values were divided by the rosette area of the same plant on the same day (Figure 3). The effect of salt stress was determined by performing an ANOVA per accession and treatment with Tukey's post hoc test of significance for each greenness hue $(p$-value $<0.05)$.

\section{Kinetic Chlorophyll Fluorescence Imaging and Processing}

To assess the effect of salt stress on photosynthetic performance, ChlF measurements were acquired using an enhanced version of the FluorCam FC-800MF pulse amplitude modulated (PAM) chlorophyll fluorometer (PSI, Czech Republic). The ChlF imaging station was mounted on a robotic arm with an LED light panel and a high-speed charge-coupled device camera (pixel resolution of $720 \times 560$, frame rate $50 \mathrm{fps}$ and 12-bit depth) positioned in the middle of the light panel (Supplementary Figure S2). The LED panel was equipped with $3 \times 64$ orange-red $(618 \mathrm{~nm})$ and $64 \mathrm{cool}-$ white LEDs $(6500 \mathrm{~K})$, distributed equally over $75 \times 75 \mathrm{~cm}$. This resulted in a $\pm 5 \%$ maximum deviation from the mean across the imaged area of $35 \times 35 \mathrm{~cm}$. Modulated light of known wavelength was applied to detect the ChlF signal. Three types of light sources were used: (1) PAM short-duration measuring flashes $(33 \mu \mathrm{s})$ at $618 \mathrm{~nm},(2)$ orange-red $(618 \mathrm{~nm})$ and cool-white $(6500 \mathrm{~K})$ actinic lights with maximum irradiance $440 \mu \mathrm{mol} \mathrm{m} \mathrm{m}^{-2} \mathrm{~s}^{-1}$ and (3) saturating cool-white light with maximum irradiance $3000 \mu \mathrm{mol} \mathrm{m} \mathrm{m}^{-2} \mathrm{~s}^{-1}$.

Plant trays were automatically loaded into the light-isolated imaging cabinet of PlantScreen ${ }^{\mathrm{TM}}$ with a top-mounted LED 
A

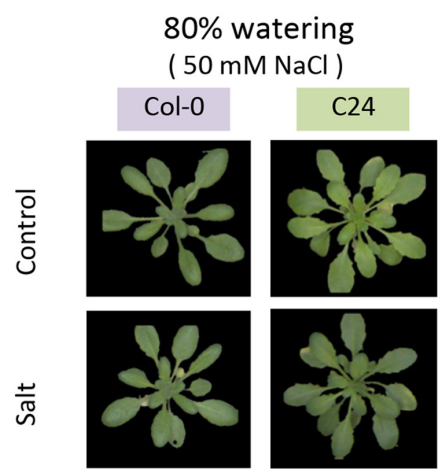

B

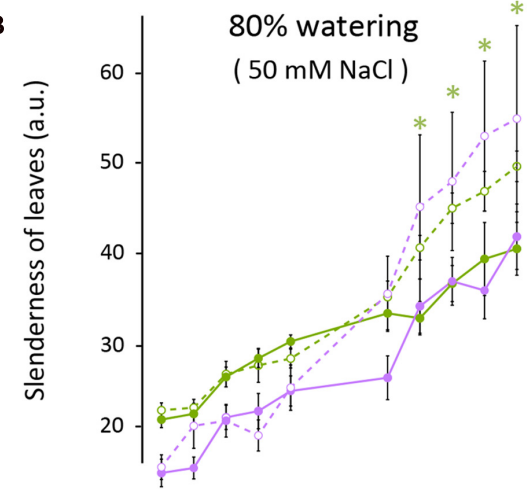

$60 \%$ watering

( $100 \mathrm{mM} \mathrm{NaCl}$ )
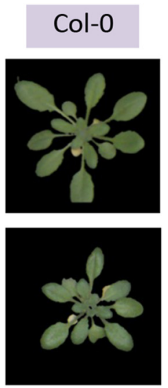

$60 \%$ watering

$(100 \mathrm{mM} \mathrm{NaCl}$ )

$\mathrm{C} 24$
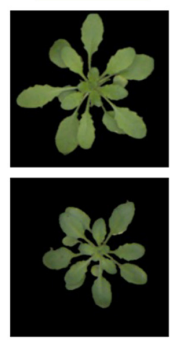

C

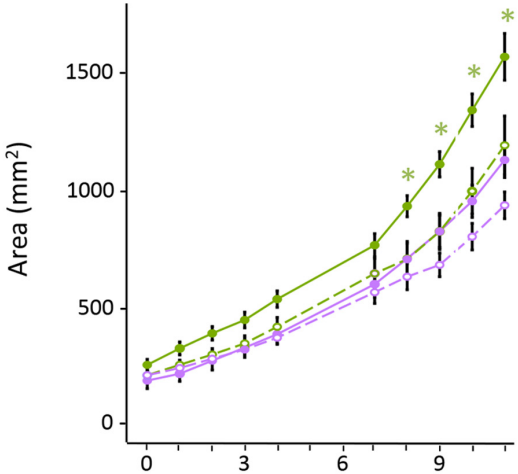

$\mathrm{SIIT}_{1}$

D

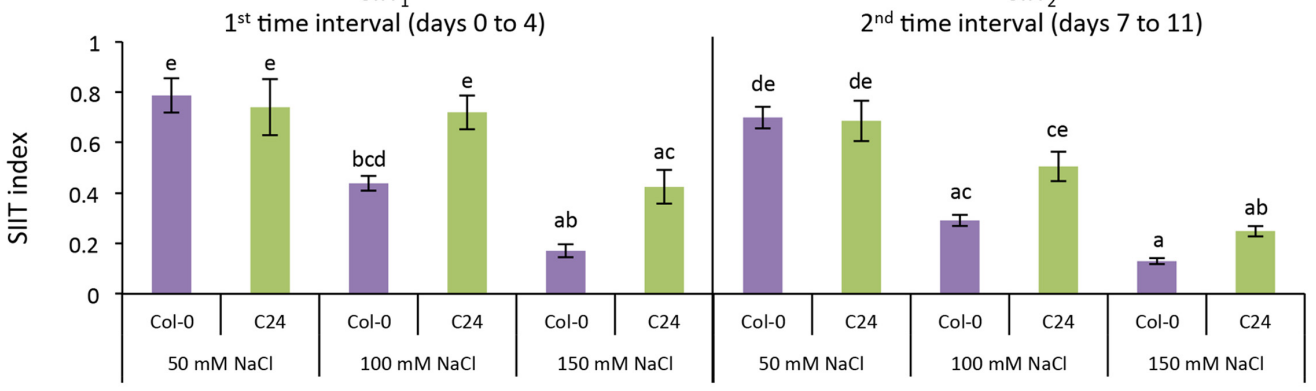

$40 \%$ watering

( $150 \mathrm{mM} \mathrm{NaCl}$ )

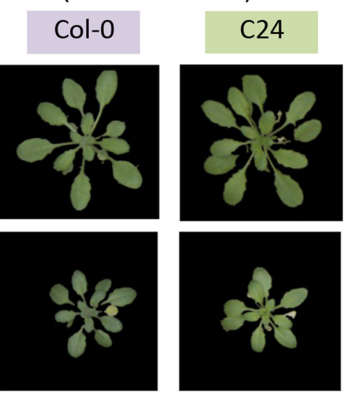

$40 \%$ watering

( $150 \mathrm{mM} \mathrm{NaCl}$ )

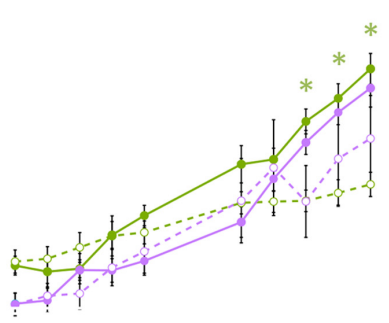

- Col-0 Control -c- C24 Control

Col-0 Salt C24 Salt
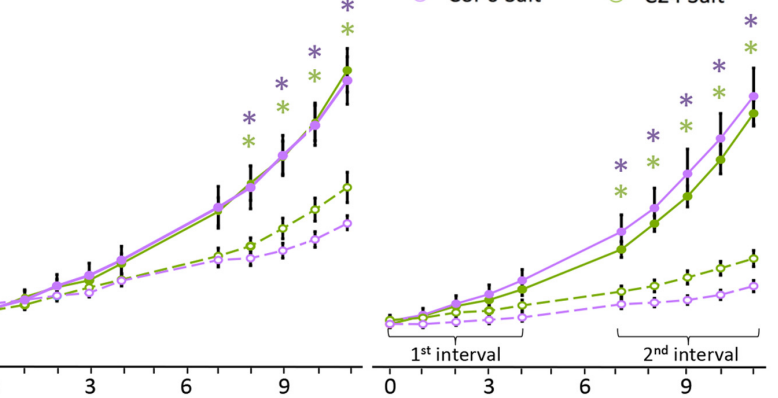

0

$\begin{array}{ccc}3 & 6 & 9 \\ \text { Days after } & \mathrm{NaCl} & \text { treatment }\end{array}$

$\mathrm{SIIT}_{2}$ 


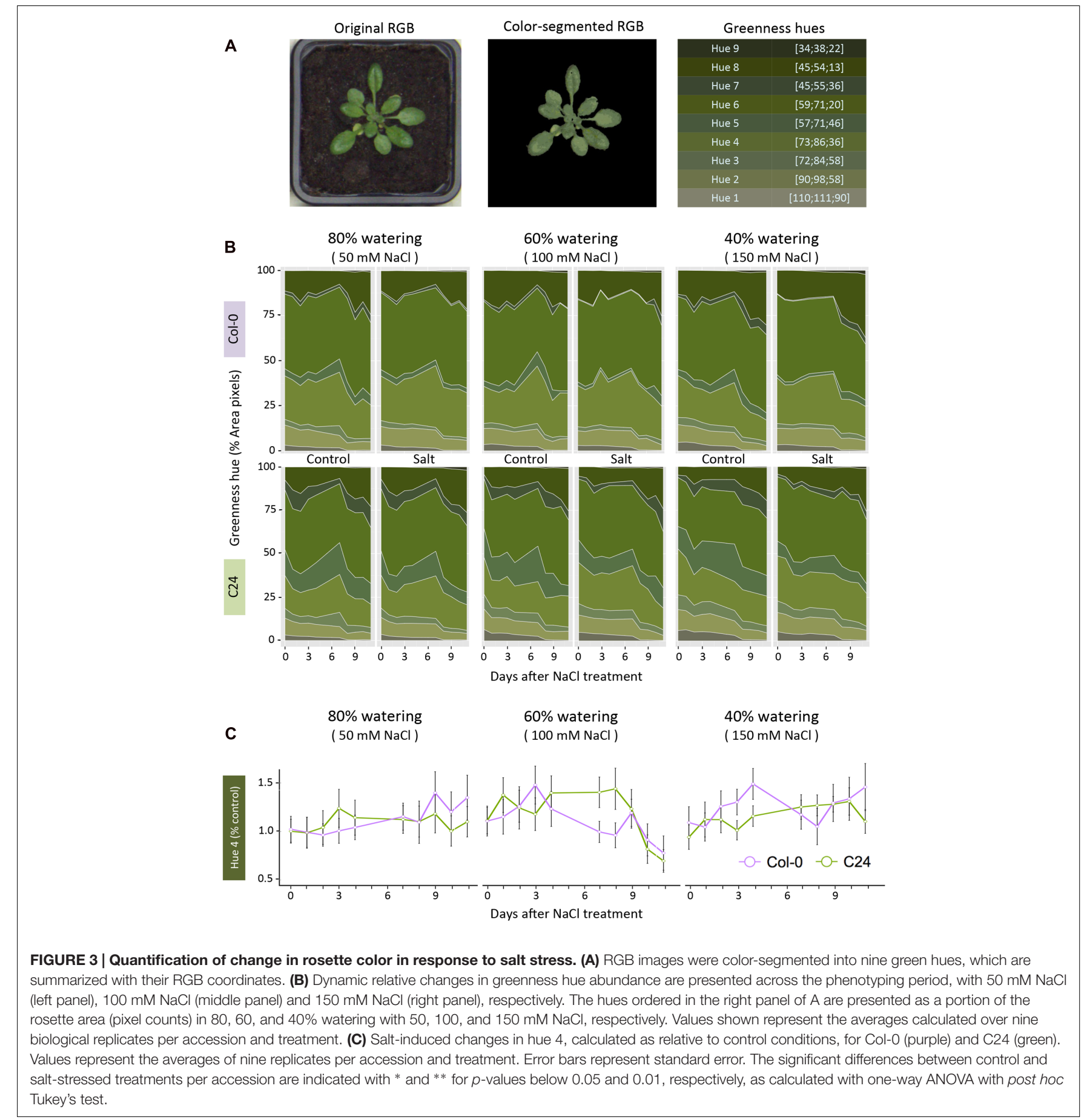

light panel. After the 15 min dark-adaptation period, when PSII reaction centers open, the trays were automatically transported to the ChlF imaging cabinet. A $5 \mathrm{~s}$ flash of light was applied to measure the minimum level of fluorescence in the darkadapted state $\left(\mathrm{F}_{\mathrm{O}}\right)$, followed by a saturation pulse of $800 \mathrm{~ms}$ (with an irradiance of $1200 \mu \mathrm{mol} \mathrm{m} \mathrm{m}^{-2} \mathrm{~s}^{-1}$ ) used to determine the maximum fluorescence in the dark-adapted state $\left(\mathrm{F}_{\mathrm{m}}\right)$. Plants were relaxed in the dark for $17 \mathrm{~s}$ and then subjected to $70 \mathrm{~s}$ of cool-white actinic lights to drive photosynthesis and measure the peak rise in fluorescence $\left(\mathrm{F}_{\mathrm{p}}\right)$. These conditions were used in both ChlF imaging techniques using quenching kinetics and light curve protocol.

For quenching kinetics protocol, additional saturation pulses were applied at $8,18,28,48,68 \mathrm{~s}$ during actinic illumination, corresponding to L1, L2, L3, L4, and Lss states at a constant photon irradiance of $210 \mu \mathrm{mol} \mathrm{m} \mathrm{m}^{-2} \mathrm{~s}^{-1}$ (Supplementary Figure S3). These ChlF signals were used to acquire the maximum fluorescence in the light-adapted state $\left(\mathrm{F}_{\mathrm{m}}{ }^{\prime}\right)$, and the 
level of $\mathrm{ChlF}$ measured just before the saturation pulse was considered the steady-state fluorescence in the light-adapted state $\left(F_{t}\right)$. Further responses to dark-relaxation were measured by switching the actinic light off for $100 \mathrm{~s}$ and applying saturating pulses at 30, 60, and $90 \mathrm{~s}$, corresponding to D1, D2, D3 states (Supplementary Figure S2). The PlantScreen ${ }^{\mathrm{TM}}$ Analyzer software performed the automated ChlF feature extraction by mask application, background subtraction and parameter calculation based on the fluorescence levels of $F_{o}, F_{m}, F_{p}$, $F_{t}$ and $F_{m}{ }^{\prime}$, which were estimated by integrating pixel-bypixel values across the entire rosette (Supplementary Figure S2; Supplementary Table S2). Minimum fluorescence in the lightadapted stated $\left(\mathrm{F}_{\mathrm{o}}{ }^{\prime}\right)$ was calculated according to Oxborough and Baker (1997).

For the examination of natural variation in the nine Arabidopsis accessions we optimized ChlF imaging by quantifying the rate of photosynthesis at different photon irradiances using the light curve protocol (Henley, 1993; Rascher et al., 2000) which was proven to provide detailed information on ChlF under stress (Brestic and Zivcak, 2013). A $5 \mathrm{~s}$ flash of light was applied to measure the minimum fluorescence, followed by a saturation pulse of $800 \mathrm{~ms}$ (with an irradiance of $1200 \mu \mathrm{mol} \mathrm{m}{ }^{-2} \mathrm{~s}^{-1}$ ) to determine the maximum fluorescence in the dark-adapted state. Next, $60 \mathrm{~s}$ intervals of cool-white actinic light at 95, 210, 320, $440 \mu \mathrm{mol} \mathrm{m} \mathrm{m}^{-2} \mathrm{~s}^{-1}$ corresponding to L1, L2, L3, and L4, respectively, were applied. A saturation pulse was applied at the end of the period of actinic light to acquire the maximal fluorescence in the lightadapted state (Supplementary Figure S6A). The ChlF signal measured just before the saturation pulse was taken as the steady-state fluorescence value in the light-adapted state. The ChlF parameters were extracted and processed as described above for data collected from day 0 to day 7 of salt treatment (Figure 6).

\section{Statistical Analysis on Chlorophyll Fluorescence-Related Responses to Salt Stress}

An ANOVA with Tukey's post hoc test of significance ( $p$ value $<0.05$ ) was used to evaluate the differences in ChlF between control and salt-stressed plants. Trait values specific to accession, day and condition were divided by the overall average per trait to analyze the fluctuations in the ChlF traits, which were due to both plant development and salt treatment (Figures 4 and 6). Principal component analysis (PCA) was performed on $20 \mathrm{ChlF}$ traits collected from Col-0 and C24 under the different adapted states and saturating pulses (L1 to L4, Lss and D1 to D3) to reduce data dimensionality (Supplementary Figure S5; Supplementary Table S3). Eight ChlF traits $\left(\mathrm{F}_{\mathrm{v}} / \mathrm{F}_{\mathrm{m}}, \mathrm{F}_{\mathrm{v}}{ }^{\prime} / \mathrm{F}_{\mathrm{m}}{ }^{\prime}, \Phi P\right.$, qP, $\Phi$ NO, $\Phi$ NPQ, qN and NPQ; Lazar, 2015) measured on day 7 at $440 \mu \mathrm{mol} \mathrm{m}{ }^{-2} \mathrm{~s}^{-1}$ were clustered with SIIT $_{1}$ and SIIT $_{2}$ $\left(\mathrm{GR}_{\text {salt }} / \mathrm{GR}_{\text {control }}\right.$ for each time interval) using the Ward linkage method. This was performed to study the relationships between the ChlF traits and relative changes in growth rate under salt stress among the nine accessions (Figure 7). Normalization was done by dividing the relative trait values by the overall average per trait. Mann-Whitney $U$-test with a continuity correction were performed on the ChlF parameters captured by the light curve protocol, and $p$-values were calculated using treatment as a grouping variable per accession for each day of the phenotyping period (Supplementary Table S4).

\section{RESULTS}

\section{Salt Stress Affected Growth and Morphology-Related Traits Over Time}

To define the most suitable screening conditions to study early responses to salt stress, we first aimed to optimize the salt treatment (Figure 1). Three watering regimes were used to control the soil-water content (40, 60, and 80\%; Figure 1A). The salt solution was diluted according to the watering regime to the final concentrations of 150,100 , or $50 \mathrm{mM} \mathrm{NaCl}$ in the soil corresponding to severe, moderate and mild salt stress, respectively (Figure 1B) and plants were phenotyped using RGB and ChlF imaging (Figure 1C). We then analyzed the phenotypes of Col-0 and C24 plants to discern the conditions most suitable for screening early salt-induced changes without greatly compromising plant health (Figure 2).

Investigation of rosette morphology revealed that Col-0 developed more slender leaves than C24 under control conditions (Figure 2B; Supplementary Table S1), but under severe salt stress, differences were less pronounced. Changes in roundness and compactness of Col-0 and C24 leaves were apparent after 23 days of salt treatment, whereas changes in rotational mass symmetry, eccentricity and slenderness of Col-0 leaves were recorded after 1 day of salt treatment (Supplementary Table S1; Figure 2C). C24 plants showed significant decreases in rosette area with salt treatment at day 8 in mild and moderate stress conditions and at day 7 in severe stress conditions. Mild stress levels did not cause a significant decrease in rosette area in Col-0 plants but a marked decrease was observable at day 8 with moderate and at day 7 with severe salt stress (Figure 2C).

Growth rates were estimated in each accession and condition by splitting the growth period into two intervals, $0-4$ days and from 7 to 11 days, and fitting two linear functions to the increase in rosette area over time (Figure 2C). Growth rates of salt-treated plants were smaller in both intervals than those of control plants. This reduction was more pronounced in the second interval (Supplementary Table S1), allowing the discrimination between early and late responses to salt stress. The ratio of $\mathrm{GR}_{\text {salt }}$ to $\mathrm{GR}_{\text {control }}$ was used to describe the SIIT index and was calculated to assess salinity tolerance in the early $\left(\mathrm{SIIT}_{1}\right)$ and late $\left(\mathrm{SIIT}_{2}\right)$ phases of salt stress (Figure 2D). There was a clear decrease in SIIT values in both Col-0 and C24 plants with increasing salt stress levels. Both $\mathrm{SIIT}_{1}$ and $\mathrm{SIIT}_{2}$ of $\mathrm{C} 24$ were higher than those of Col-0 under moderate and severe salt stress conditions in both intervals, indicating that C24 has higher salinity tolerance. Differences between control and salt-stressed plants and between Col-0 and C24 were most pronounced under moderate and severe conditions of salt stress. In addition, control plants grown in the $40 \%$ soil-water content were much smaller than control plants grown in other watering regimes (Figure 2C), suggesting that these plants were likely to be suffering from drought stress. Mild salt stress had no effect on the growth of Col-0 and only 

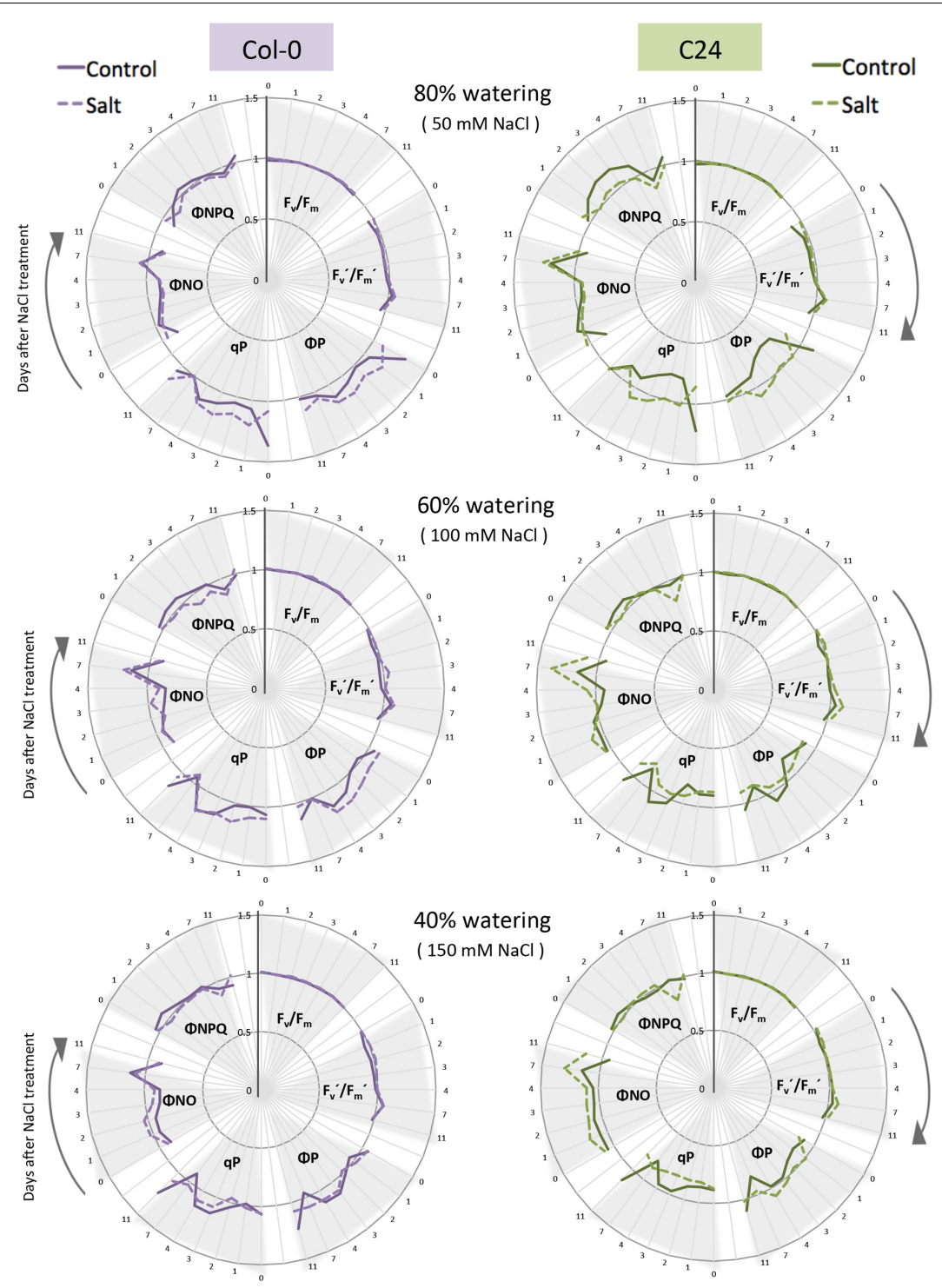

FIGURE 4 | The effect of watering regime and salt treatment on ChIF parameters. Maximum quantum yield of PSII photochemistry for the dark-adapted $\left(F_{v} / F_{m}\right)$ and light-adapted $\left(F_{v}^{\prime} / F_{m}{ }^{\prime}\right)$ states, the quantum yield of PSIl photochemistry for the light-adapted state $(\Phi P)$, the photochemical quenching coefficient that estimates the fraction of open PSII reaction centers (qP), the quantum yield of constitutive non-regulatory non-photochemical dissipation processes ( $\Phi$ NO) and the quantum yield of regulatory non-photochemical quenching ( $\Phi$ NPQ) were measured using the quenching protocol for Col-0 (purple) and C24 (green) plants in control (solid line) and salt-stressed (dashed line) conditions. Time in days is indicated on the outer rim of the graph. Individual ChIF traits are presented in clusters indicated by gray triangles. Values represent the average of nine biological replicates per accession and treatment divided by the overall average per trait. Values were measured for plants grown in 80,60 , and $40 \%$ soil-water contents in control or salt-stressed conditions.

a slight effect was observed later in C24 plants (Figure 2B). We established that plant growth and performance was best assessed using a $60 \%$ watering regime, which resulted in moderate salt stress of $100 \mathrm{mM} \mathrm{NaCl}$.

\section{Color Segmentation of RGB Images Illustrates Changes in Rosette Greenness Over Time}

We examined the features of pixel color in the RGB images to identify changes in rosette greenness under salt stress. Color information was extracted from pixels corresponding to the imaged rosettes. RGB images were color-segmented into nine green hues and analyzed for their relative abundance as a percentage of rosette area (Figure 3A). This strategy enabled the number of pixels representing each hue to be normalized for rosette area and compared between accessions and treatments (Figure 3B). Hues 1, 2, 5, and 8 changed over time without marked differences between treatments, while hues 3, 4, 6, 7, and 9 differed from control conditions after only 1 day of exposure to salt stress (Figure 3B). We calculated the ratio for each hue, between control and salt-treated plants, to observe 

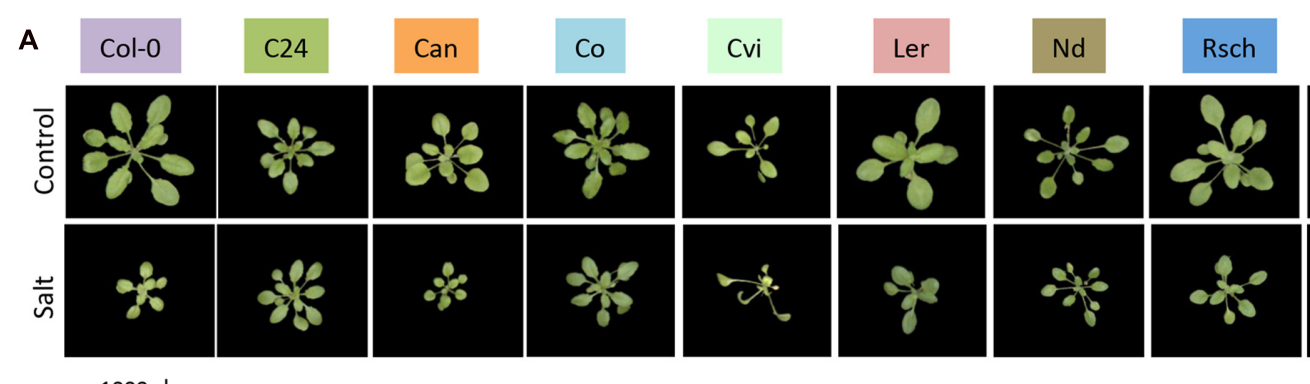

$\mathrm{Te}$

B
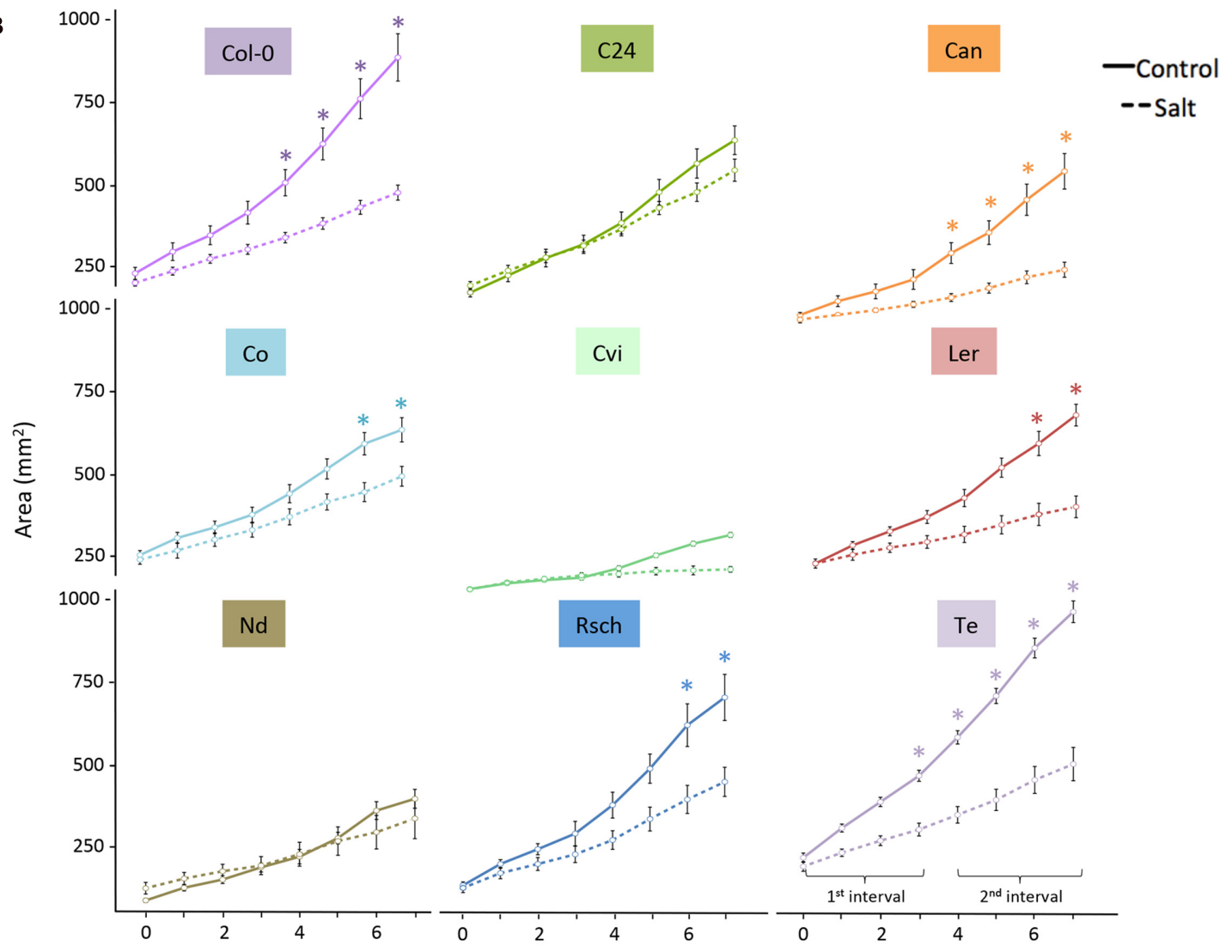

C

$\mathrm{SIIT}_{1}$
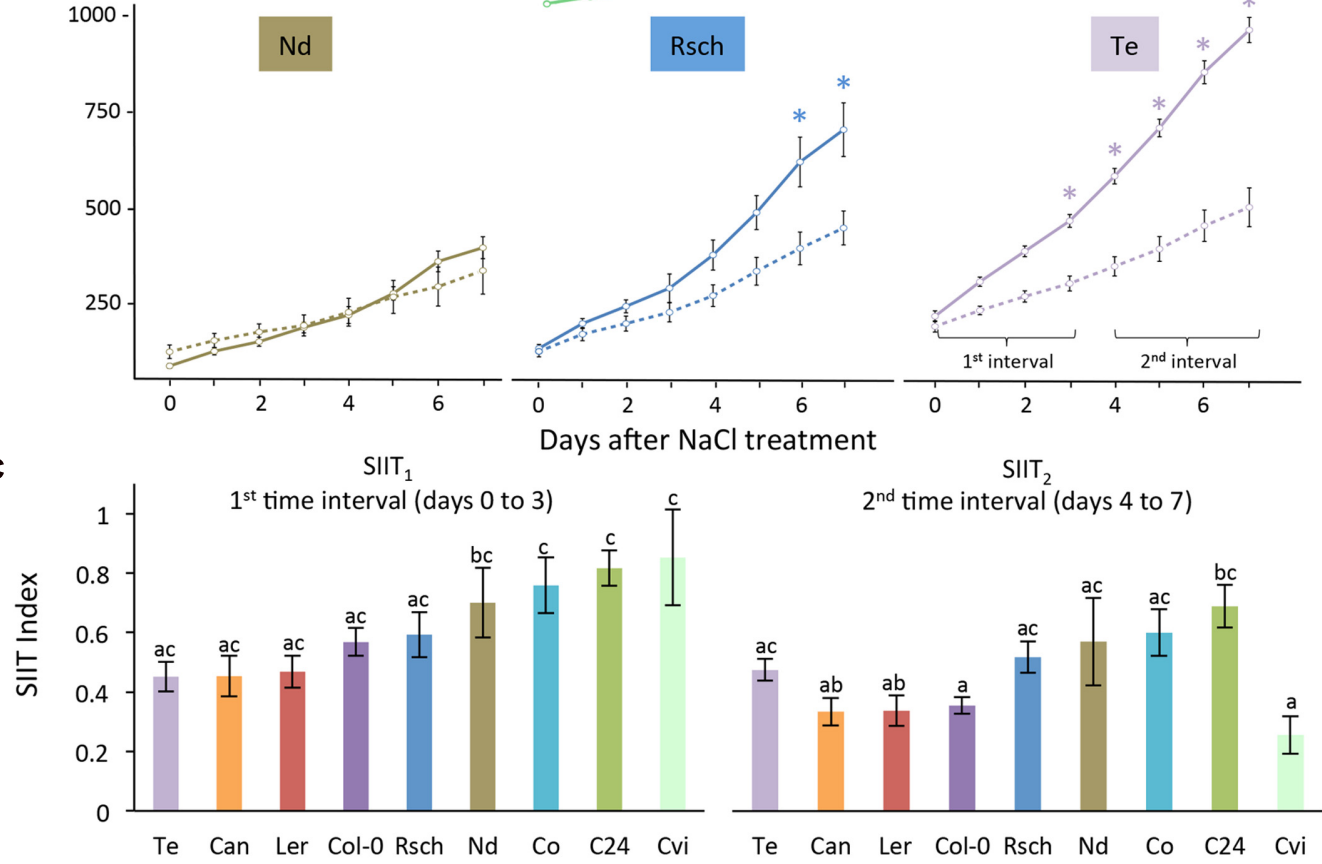

$\mathrm{SIIT}_{2}$

$2^{\text {nd }}$ time interval (days 4 to 7 )

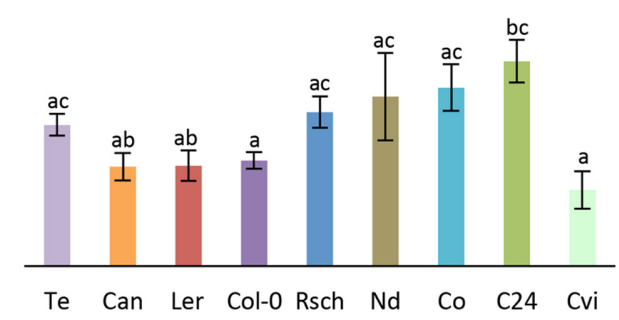

FIGURE 5 | Natural variation in growth-related responses of nine Arabidopsis accessions under salt stress. (A) RGB images of control (upper panel) and 7 days salt-stressed (lower panel) plants. (B) Projected rosette area over time for plants grown in control (solid lines) and salt-stressed (dashed lines) conditions. Values represent the average of eight biological replicates per accession and treatment. Error bars represent standard error. Significant differences between control and salt stress treatment are indicated with * and ** for $p$-values below 0.05 and 0.01 , respectively. (C) Shoot ion-independent tolerance (SIIT ${ }_{1}$ and SIIT $T_{2}$ ) values were calculated from averages of eight replicates per accession and treatment. Error bars represent standard error. Different letters indicate significant differences between accessions as tested with one-way ANOVA with post hoc Tukey's test $(p<0.05)$. 

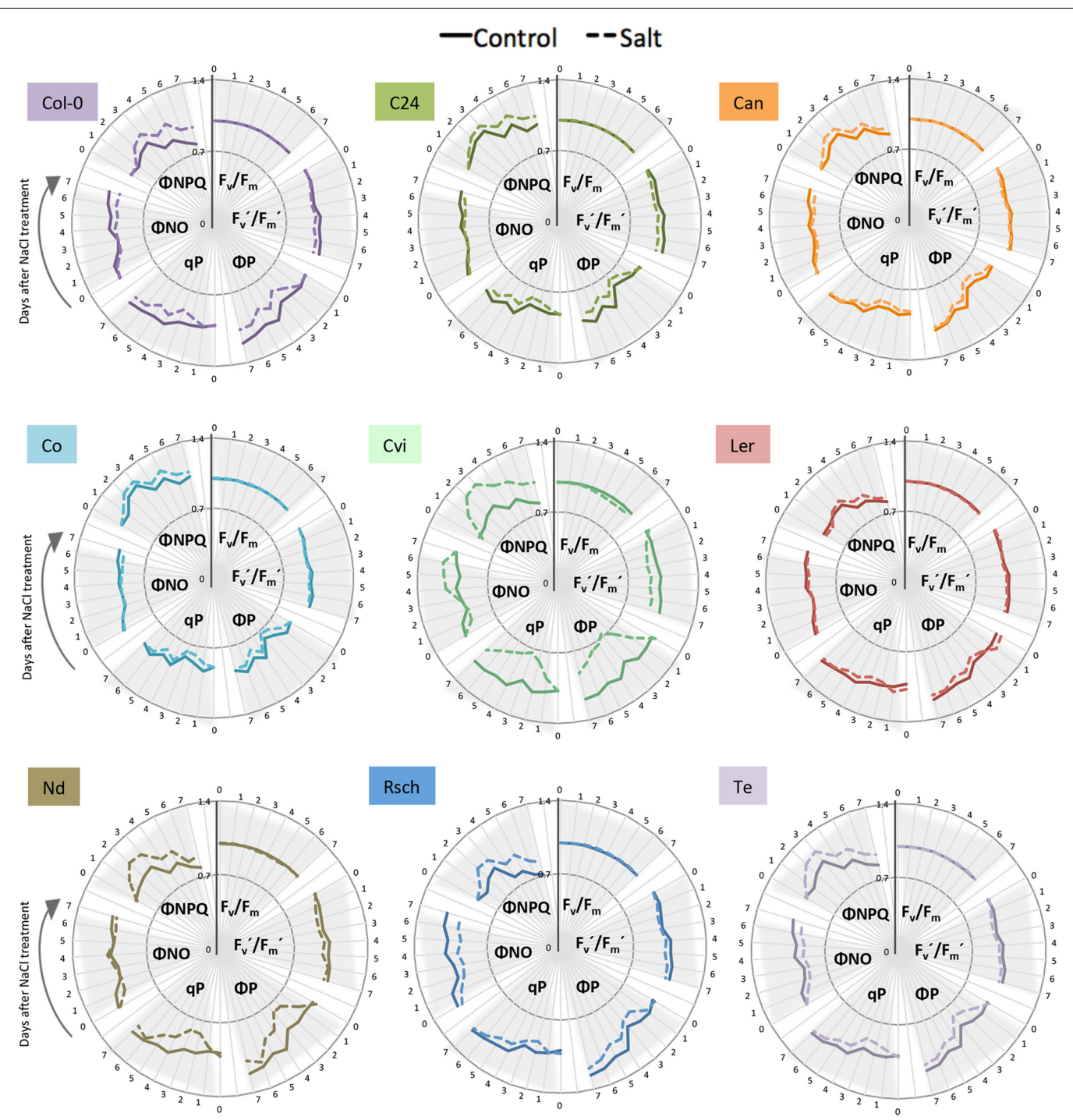

FIGURE 6 | Natural variation in ChIF-related responses of nine Arabidopsis accessions under salt stress. Maximum quantum yield of PSII photochemistry for the dark-adapted $\left(F_{v} / F_{m}\right)$ and light-adapted $\left(F_{v}^{\prime} / F_{m}{ }^{\prime}\right)$ states, the quantum yield of PSIl photochemistry for the light-adapted state $(\Phi P)$, the photochemical quenching coefficient that estimates the fraction of open PSII reaction centers (qP), the quantum yield of constitutive non-regulatory non-photochemical dissipation processes $(\Phi \mathrm{NO})$ and the quantum yield of regulatory non-photochemical quenching $(\Phi \mathrm{NPQ})$ measured by the light curve protocol; control (solid line) and salt-stressed (dashed line) conditions. Time in days is indicated on the outer rim of the graph. Individual ChIF traits are presented in clusters indicated by gray triangles. Values represent the average of eight replicates per accession and treatment divided by the overall average per trait.

the salt-induced changes among between the accessions and treatments. We presented the results for hue 4, showcasing the differences between Col-0 and C24 throughout the phenotyping period (Figure 3C).

\section{Chlorophyll Fluorescence Imaging Captures the Early and Late Changes in Photosynthetic Performance in Response to Salt Stress}

To further explore the photosynthetic performance of control and salt-treated plants, we used ChlF parameters measured by the PAM method and quenching kinetics. From the measured fluorescence transient states, the basic ChlF parameters were derived (i.e., $F_{o}, F_{m}, F_{m}{ }^{\prime}, F_{t}, F_{v}$, and $F_{p}$ ), which were used to calculate the quenching coefficients (i.e., qP, NPQ, PQ, and $\mathrm{qN})$ and other parameters characterizing plant photosynthetic performance (i.e., $\mathrm{F}_{\mathrm{o}}{ }^{\prime}, \mathrm{F}_{\mathrm{v}} / \mathrm{F}_{\mathrm{m}}, \Phi P, \mathrm{~F}_{\mathrm{v}}{ }^{\prime} / \mathrm{F}_{\mathrm{m}}{ }^{\prime}$, $\Phi \mathrm{NO}$, $\Phi \mathrm{NPQ}$ and Rfd; these parameters are summarized in Supplementary Table S2). The quenching kinetics protocol allowed to detect the shifts in the ChlF curves of Col-0 and C24 salt-stressed plants as early as $1 \mathrm{~h}$ after moderate or severe stress (Supplementary Figure S3).

We then selected six ChlF parameters that reflect the photosynthetic function of PSII (Lazar, 2015): the maximum quantum yield of PSII photochemistry in the dark-adapted $\left(\mathrm{F}_{\mathrm{V}} / \mathrm{F}_{\mathrm{m}}\right)$, and the light-adapted $\left(\mathrm{F}_{\mathrm{v}}{ }^{\prime} / \mathrm{F}_{\mathrm{m}}{ }^{\prime}\right)$ states, the coefficient 
A

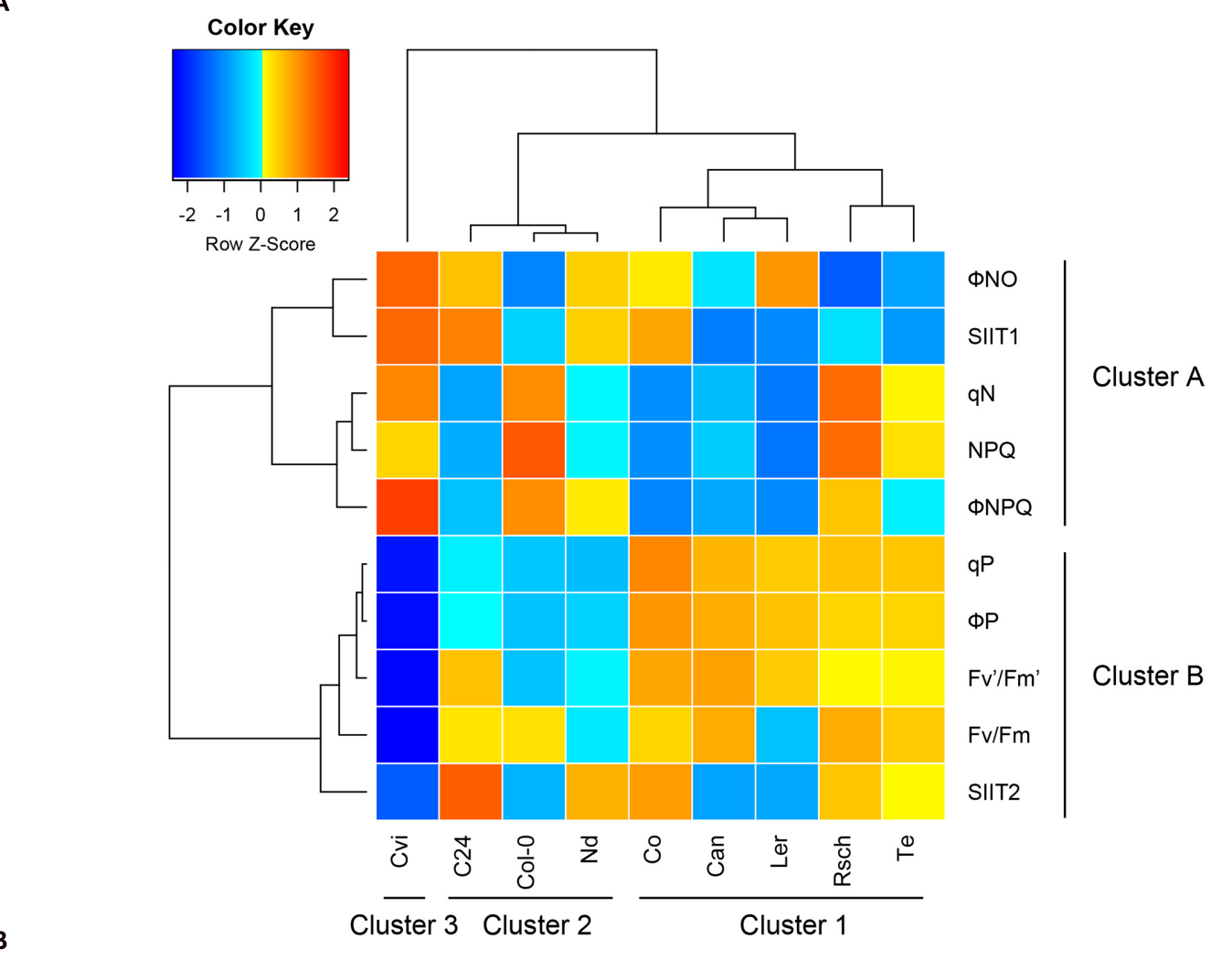

B

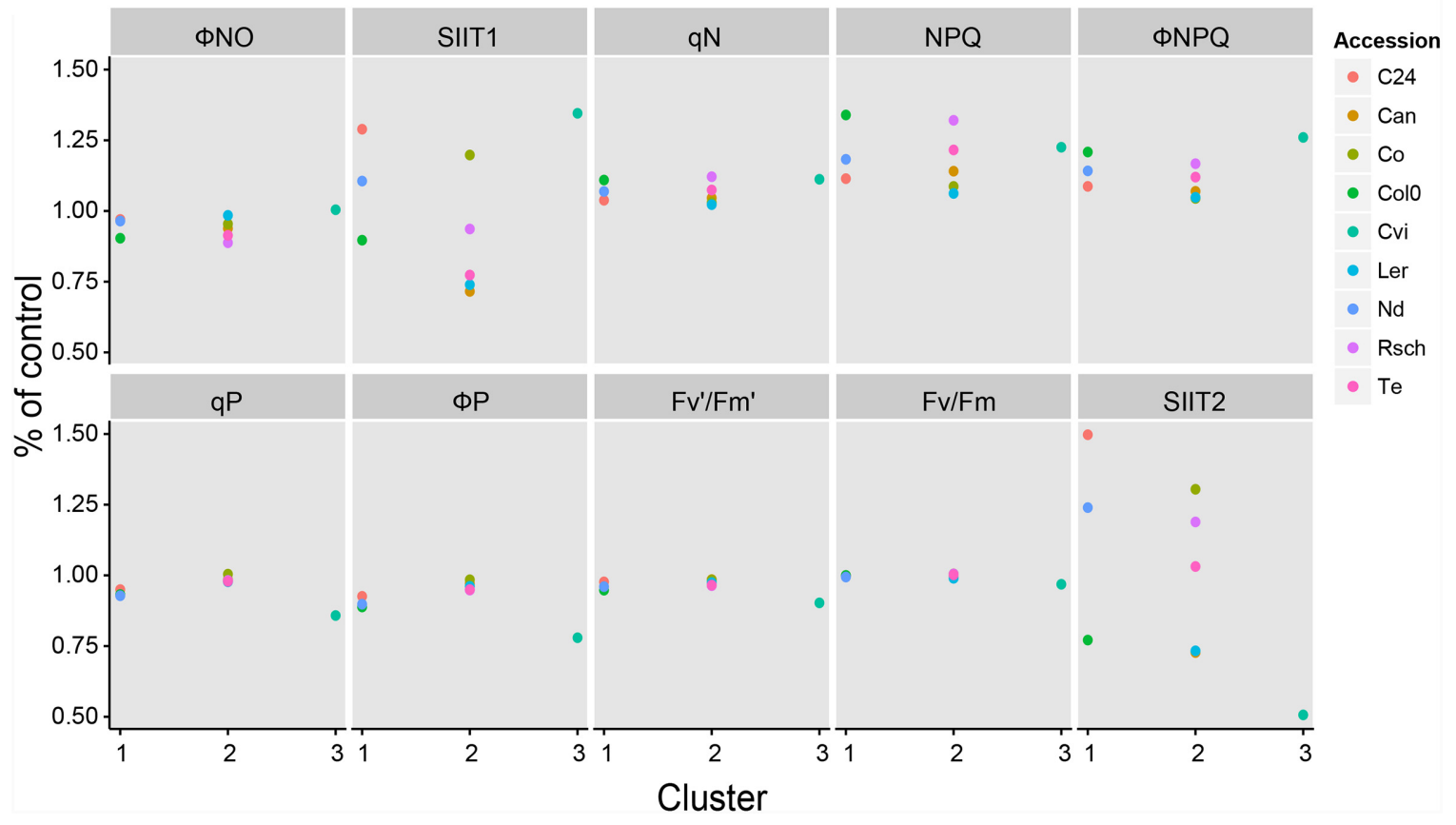

FIGURE 7 | Clustering of ChIF parameters with SIIT values revealed the early and late responses to salt stress. (A) Using the Ward Linkage method, the shoot-ion independent tolerance (SIIT) values, SIIT, SIIT 2 and eight ChIF traits, measured at the highest photon irradiance (L4) at day 7 , were clustered. Three cluster groups were identified for the nine accessions, while two clusters were observed to form across the phenotypic traits. The trait values for individual accessions presented in the heat map were normalized by the z-Fisher transformation per trait. (B) The effect of salt treatment on ChIF parameters and SIIT values across the three cluster groups identified in (A). SIIT 1 , SIIT 2 and the selected ChIF parameters were calculated relative to control conditions and divided by the overall average per trait. Values represent average of eight biological replicates per accession and treatment. 
of photochemical quenching that estimates the fraction of open PSII reaction centers $(\mathrm{qP})$, the actual quantum yield of PSII photochemistry in the light-adapted state, the proportion of light absorbed by the chlorophyll associated with PSII that is used in photochemistry $(\Phi P)$, the quantum yield of constitutive non-light-induced dissipation consisting of ChlF emission and heat dissipation ( $\Phi N O)$ and the quantum yield of regulatory light-induced heat dissipation ( $\Phi$ NPQ) for days $0,1,2,3$, 4,7 , and 11 of the phenotyping period (Figure 4 ). The traits related to maximum quantum yield $\left(\mathrm{F}_{\mathrm{v}} / \mathrm{F}_{\mathrm{m}}\right.$ and $\left.\mathrm{F}_{\mathrm{v}}{ }^{\prime} / \mathrm{F}_{\mathrm{m}}{ }^{\prime}\right)$ were not significantly different between control and salt-treated plants (Figure 4; Supplementary Figure S4; Supplementary Table S2), suggesting that PSII was not damaged during the course of the experiment. The other four ChlF parameters (qP, ФP, ФNO, and $\Phi N P Q)$ varied with time in both control and salt-stressed conditions (Figure 4).

Change in nine ChlF parameters, with respect to control conditions, was used to indicate the effect of salt stress on quenching processes and PSII efficiency (Supplementary Table S2; Supplementary Figure S4): traits measured in the lightadapted state were most affected (L1, L2, L3, L3, and Lss), while traits measured in the dark-adapted state (D1, D2, and D3) did not vary between treatments or accessions, except in the case of NPQ, qN, RFD, and $\Phi$ NPQ under severe stress (Supplementary Figure $\mathrm{S} 4) . \mathrm{F}_{\mathrm{v}}{ }^{\prime} / \mathrm{F}_{\mathrm{m}}{ }^{\prime}$ was unchanged in all salt treatments and in both accessions and $\Phi N O$ displayed only slight changes indicating that those parameters were robust in response to salt stress. With this protocol we were able to detect rapid changes in $\Phi P, \mathrm{qP}, \mathrm{PQ}, \mathrm{NPQ}, \mathrm{qN}, \mathrm{RFD}$, and $\Phi$ NPQ in C24, but not in Col-0, after only 1 day of salt treatment (Supplementary Figure S4). To explore the ChlF parameters even further, we used PCA to classify the observed trends (Supplementary Figure S5). PCA performed on the $20 \mathrm{ChlF}$ traits, under the eight different adapted states and saturating pulses (L1 to L4, Lss and D1 to D3), showed that the five PCs explained $85 \%$ of the variation (Supplementary Figure S5). PC1 described accession-specific trends and PC2 contained traits relevant to treatment with salt (Supplementary Table S3).

\section{Natural Variation of Growth-Related Traits was Quantified in Response to Salt Stress}

Because moderate salt stress elicited significant changes between control and salt-treated plants without symptoms of severe stress, it was used to investigate natural variation among the nine accessions of Arabidopsis thaliana (Figure 5A). Plants were cultivated with a longer light period at higher temperatures and lower photon irradiance than the initial conditions for Col-0 and C24. Phenotyping of the plants was conducted in the same manner through 7 days after salt treatment using RGB (Figure 5A) and ChlF imaging with the light curve protocol (Supplementary Figure S6). The rosette area of Te was the most significantly reduced by salt stress starting from day 3, while Col-0, Can, Co and Ler showed significant reductions later. Rosette areas of $\mathrm{C} 24, \mathrm{Nd}$, and Cvi were not significantly reduced (Figure 5B). Natural variation was evident across all SIIT values in both intervals, indicating differences in salinity tolerance among the nine accessions. Lower SIIT $_{2}$ than SIIT $_{1}$ values indicated that plants became less tolerant over time; however, this difference was only significant for Cvi. This demonstrates that we were able to assess natural variation, not only in the growth reduction magnitude, but also in the timing of the responses to salt stress.

\section{Light Curve Chlorophyll Fluorescence Imaging Captured Early Responses to Salt Stress}

ChlF parameters were measured at four photon irradiances and were calculated as described previously (Supplementary Table S2). Differences in most ChlF parameters between control and salt-stressed plants were observed within $24 \mathrm{~h}$ of introducing salt at the highest photon irradiance (Figure 6). We found that the maximum quantum yield of photosynthesis in the darkadapted state $\left(\mathrm{F}_{\mathrm{v}} / \mathrm{F}_{\mathrm{m}}\right)$ was not affected by salt stress in Col-0. Rapid responses to salt stress were observed in photochemical and non-photochemical quenching, as $\Phi$ NPQ rapidly increased, followed by a decrease in non-regulatory heat dissipation $\Phi$ NO. The increase in heat dissipation via xanthophyll-mediated nonphotochemical quenching ( $\Phi$ NPQ) coincided with a significant decrease in the photochemical quenching coefficient $(\mathrm{qP})$ and inhibition of the PSII operating efficiency $(\Phi P)$. Maximum quantum yield in the light-adapted state $\left(\mathrm{Fv}^{\prime} / \mathrm{Fm}^{\prime}\right)$ decreased in response to salt stress, but not as severely as did the other ChlF traits (Supplementary Figure S6B; Supplementary Table S4).

Because the highest actinic photon irradiance (L4) provided the most discriminative power for the quantification of early saltinduced changes in ChlF parameters, it was chosen to assess the natural variation in photosynthetic activity. Comparison among the nine accessions using the six key ChlF traits revealed that $\mathrm{F}_{\mathrm{v}} / \mathrm{F}_{\mathrm{m}}$ did not differ between control and salt-treated plants, with the exception of Cvi (Figure 6; Supplementary Table S4). Increased $\Phi \mathrm{NPQ}$, upon exposure to salt stress, was observed to varying degrees (Figure 6; Supplementary Table S4). We also observed natural variation in the salt-induced decrease of $\Phi P$, $\mathrm{qP}$, and $\Phi N O$ over time. Overall, we observed that salt stress results in rapid and substantial increase in non-photochemical processes (i.e., the dissipation of heat in the PSII antennae), which correlates with reduced PSII quantum efficiency and photochemical quenching under stress (Figure 6; Supplementary Figure S6C).

Eight ChlF traits and two SIIT values were used to cluster the nine accessions using the Ward linkage method (Figure 7A). The accessions clustered into three groups (1-3) while the phenotypic traits were classified into two clusters (A and B). Clustering of traits revealed that non-photochemical quenching-related parameters (NPQ, $\Phi \mathrm{NO}, \Phi \mathrm{NPQ}$, and $\mathrm{qN}$ ) are more prominent during the early stage (cluster $\mathrm{A}$ ), while quantum yield-related parameters $\left(\mathrm{F}_{\mathrm{v}} / \mathrm{F}_{\mathrm{m}}, \mathrm{F}_{\mathrm{v}}{ }^{\prime} / \mathrm{F}_{\mathrm{m}}{ }^{\prime}, \Phi \mathrm{P}\right.$, and $\left.\mathrm{qP}\right)$ corresponded to the later stage of exposure to salt stress (cluster B). Eight accessions were grouped into two clusters (clusters 1 and 2) and distinct responses of Cvi placed it separately (cluster 
3; Figure 7). Accessions in cluster 2 (C24, Nd, and Col-0) showed the least pronounced responses to salt stress during the early phase of exposure to salt stress in terms of $\mathrm{SIIT}_{1}$ (Figure 5C) and significant decline in photosynthetic activity $(\Phi P$ and qP; Figures 6 and 7; Supplementary Table S4). Finally, the accessions belonging to cluster 1 (Rsch, Te, Ler, Can, and Co) were characterized by rapid reduction in growth rate in the early phase of salt stress (Figure 5C) and less prominent changes in ChlF parameters (Figures 6 and 7; Supplementary Table S4). Hence, using ChlF parameters and SIIT values, we were able to distinguish between the processes affected in the early and late responses of plants to salt stress.

\section{DISCUSSION}

Recent advances in high-throughput phenotyping have allowed the parallel screening of multiple quantitative traits measuring plant growth and performance under stress conditions. In this study, we used RGB and ChlF measures, with rosette coloring, to dissect the complex responses of plants to salt stress. We developed a phenotyping protocol to monitor early physiological changes in response to salt stress involving growth, rosette morphology and photosynthetic performance. To make these evaluations, we determined the RGB, greenness and ChlF traits most responsive to salinity. The $60 \%$ soil-water content $(100 \mathrm{mM}$ $\mathrm{NaCl}$ ) was the most suitable condition for studying early plant responses to salt stress without causing growth arrest (Figure 2) or premature leaf senescence (Figure 3 ).

Investigation of the RGB images revealed that salt stress caused little change in rosette morphology (Figure 2B; Supplementary Table S1); however, these phenotypes should not be overlooked as leaf slenderness and rosette compactness are known to play important roles in heat dissipation and transpiration rate (Bridge et al., 2013). Similarly, differences in rosette greenness were predominantly related to development and accession rather than to treatment with salt (Figure 3). Nevertheless, we did observe an increase in darker hues in plants grown in severe and moderate salt stress conditions than in those grown in mild stress, which could be due to accumulation of anthocyanin (Van Oosten et al., 2013). Pronounced changes in both lighter and darker hues were previously reported to occur in the later phases of salt stress response than the phenotyping period used in this study (Ben Abdallah et al., 2016). Therefore, color segmentation and quantification of green hues could provide valuable information regarding plant development and stress-related responses, especially when combined with quantitative pigment-content analysis.

We used automated image-processing pipelines to examine the salt-induced changes in rosette area over time by fitting a linear function to describe the growth over time (Figures 2C and 5B; Supplementary Table S1). We used SIIT 1 and $\mathrm{SIIT}_{2}$ as indicators of plant salinity tolerance in two time intervals (Figures 2C and 5B) finding lower $\mathrm{SIIT}_{2}$ than $\mathrm{SIIT}_{1}$ values all accessions studied. The $\mathrm{SIIT}_{2}$ values of Cvi decreased dramatically, potentially due to its early increase in non-photochemical processes, which are represented by the traits NPQ, $\Phi \mathrm{NPQ}$ and $\mathrm{qN}$, with concurrent decreases in photochemical efficiency, represented by the traits $\mathrm{F}_{\mathrm{v}}{ }^{\prime} / \mathrm{F}_{\mathrm{m}}{ }^{\prime}$, $\Phi \mathrm{P}$, and $\mathrm{qP}$. These steps are usually followed by increase in constitutive non-light-induced dissipation ( $\Phi N O)$ and a drop in the maximal quantum efficiency of PSII in dark-adapted state $\left(\mathrm{F}_{\mathrm{v}} / \mathrm{F}_{\mathrm{m}}\right.$; Mishra et al., 2012). The observed trend of lower SIIT $_{2}$ values could be due to accumulation of ions in photosynthetic tissues, putting additional constraint on photosynthesis and subsequently plant growth (Munns and Tester, 2008).

Although, maximum quantum yield was commonly used for assessing plant performance under stress (Jansen et al., 2009; Bresson et al., 2015), we found that $F_{v} / F_{m}$ seems to be a robust parameter (Figures 4 and 6), being affected only under severe stress and not reflecting early salt stress responses, which is in agreement with previous reports (Baker and Rosenqvist, 2004). Other parameters quantifying photochemical and non-photochemical processes displayed more dynamic responses to salt stress (Figures 4 and 6). To identify the ChlF traits most responsive to salt stress, we performed PCA resulting in extracting $\mathrm{PC} 1$ and $\mathrm{PC} 2$, corresponding to the differences observed between treatment and accession, respectively (Supplementary Figure S5). According to PC1, $\mathrm{F}_{\mathrm{O}}$, $F_{m}, F_{v}, F_{t}$, and $F_{p}$ were the traits that changed the most in response to salt treatment. Based on traits contained in PC2, we found that the differences observed in $F_{v} / F_{m}, N P Q, q N$, and $\mathrm{qP}$ were more accession-specific (Supplementary Table S3). Based on our results, early $\Phi \mathrm{NPQ}, \mathrm{qP}$, and $\Phi \mathrm{P}$ responses to salt stress could be used to distinguish salt-tolerant from salt-sensitive plants (Figures 4 and 6). Similar results have been found for studies of drought and salt stress (Baker and Rosenqvist, 2004; Stepien and Johnson, 2009; Mishra et al., 2012). In conclusion, we observed largely different ChlF responses to salt stress among the accessions, evidencing that different accessions use different strategies to tolerate salt stress.

In this study, we demonstrated that phenotyping multiple quantitative traits in one experimental setup can provide new insights into the dynamics of plant responses to stress. These traits can be used to assess plant natural variation and to cluster accessions based on the magnitude and timing of their response to stress (Figure 7). Our work identified a set of phenotypes that can serve as markers for early responses to salt stress. These phenotypic markers can be used to study mutant populations, natural diversity panels and responses to other stress conditions, such as drought, cold or nutrient-deficiency, which would reveal the scope of their influence on tolerance to stress. Integrating thermal imaging into the phenotyping pipeline, along with quantifying water-use and transpiration-use efficiency, would provide a more comprehensive understanding of plant responses and development under stress. The protocol presented here can also be used to study non-model plants and crop species with more complicated 3D morphology, ultimately capturing a broad range of phenotypic traits. These traits could then be used in combination with forward genetics studies to identify genes underlying early responses to salt stress with the goal of providing new target genes for crop improvement. 


\section{AUTHOR CONTRIBUTIONS}

MA performed the optimization protocol at PSI (Czech Republic) and most of the data analyses. MA, MJ, KP, and AN wrote the manuscript. With support from DS, AN performed the natural variation experiment at PSI. MA, MJ, and JF performed the phenotypic and statistical analyses. KP selected the nine accessions of Arabidopsis thaliana and designed the natural variation experiment. MTe, SN, and SS contributed to the original concept of the project and supervised the study. MTe, MTr, and KP conceived of the project and its components. All authors discussed the results and contributed to the manuscript.

\section{FUNDING}

The research reported in this publication was supported by funding from King Abdullah University of Science and Technology (KAUST) and from the European Union's Seventh

\section{REFERENCES}

Baker, N. R. (2008). Chlorophyll fluorescence: a probe of photosynthesis in vivo. Annu. Rev. Plant Biol. 59, 89-113. doi: 10.1146/annurev.arplant. 59.032607.092759

Baker, N. R., and Rosenqvist, E. (2004). Applications of chlorophyll fluorescence can improve crop production strategies: an examination of future possibilities. J. Exp. Bot. 55, 1607-1621. doi: 10.1093/jxb/erh196

Ben Abdallah, S., Aung, B., Amyot, L., Lalin, I., Lachaal, M., Karray-Bouraoui, N., et al. (2016). Salt stress $(\mathrm{NaCl})$ affects plant growth and branch pathways of carotenoid and flavonoid biosyntheses in Solanum nigrum. Acta Physiol. Plant. 38:72. doi: 10.1007/s11738-016-2096-8

Berger, B., de Regt, B., and Tester, M. (2012). Trait dissection of salinity tolerance with plant phenomics. Methods Mol. Biol. 913, 399-413. doi: 10.1007/978-161779-986-0_27

Bresson, J., Vasseur, F., Dauzat, M., Koch, G., Granier, C., and Vile, D. (2015). Quantifying spatial heterogeneity of chlorophyll fluorescence during plant growth and in response to water stress. Plant Methods 11:23. doi: 10.1186/s13007-015-0067-5

Brestic, M., and Zivcak, M. (2013). "PSII fluorescence techniques for measurement of drought and high temperature stress signal in crop plants: protocols and applications," in Molecular Stress Physiology of Plants, eds R. G. Rout and B. A. Das (New Delhi: Springer), 87-131.

Bridge, L. J., Franklin, K. A., and Homer, M. E. (2013). Impact of plant shoot architecture on leaf cooling: a coupled heat and mass transfer model. J. R. Soc. Interface 10:20130326. doi: 10.1098/rsif.2013.0326

Brown, T. B., Cheng, R., Sirault, X. R., Rungrat, T., Murray, K. D., Trtilek, M., et al. (2014). Traitcapture: genomic and environment modelling of plant phenomic data. Curr. Opin. Plant Biol. 18, 73-79. doi: 10.1016/j.pbi.2014.02.002

Cabot, C., Sibole, J. V., Barcelo, J., and Poschenrieder, C. (2014). Lessons from crop plants struggling with salinity. Plant Sci. 226, 2-13. doi: 10.1016/ j.plantsci.2014.04.013

Campbell, M. T., Knecht, A. C., Berger, B., Brien, C. J., Wang, D., and Walia, H. (2015). Integrating image-based phenomics and association analysis to dissect the genetic architecture of temporal salinity responses in rice. Plant Physiol. 168, 1476-1489. doi: 10.1104/pp.15.00450

Chaves, M. M., Flexas, J., and Pinheiro, C. (2009). Photosynthesis under drought and salt stress: regulation mechanisms from whole plant to cell. Ann. Bot. 103, 551-560. doi: 10.1093/Aob/Mcn125

Chen, T. W., Kahlen, K., and Stutzel, H. (2015). Disentangling the contributions of osmotic and ionic effects of salinity on stomatal, mesophyll, biochemical
Framework Program for research, technological development and demonstration under grant agreement no GA-2013-608422 IDP BRIDGES.

\section{ACKNOWLEDGMENTS}

We would like to thank Radka Mezulaniková for assisting with the optimization protocol, Kumud Mishra (CzechGlobe, Czech Republic) for providing the Arabidopsis thaliana seeds and Dušan Lazár for comments on the ChlF analysis. We also thank Carolyn Unck for reviewing and editing the manuscript and Dr. Xavier Sirault for suggesting the use of the saturation method for salt treatment.

\section{SUPPLEMENTARY MATERIAL}

The Supplementary Material for this article can be found online at: http://journal.frontiersin.org/article/10.3389/fpls.2016.01414

and light limitations to photosynthesis. Plant Cell Environ. 38, 1528-1542. doi: 10.1111/pce. 12504

Dhondt, S., Wuyts, N., and Inze, D. (2013). Cell to whole-plant phenotyping: the best is yet to come. Trends Plant Sci. 18, 433-444. doi: 10.1016/J.Tplants.2013.04.008

Fricke, W., Akhiyarova, G., Wei, W. X., Alexandersson, E., Miller, A., Kjellbom, P. O., et al. (2006). The short-term growth response to salt of the developing barley leaf. J. Exp. Bot. 57, 1079-1095. doi: 10.1093/Jxb/Erj095

Ghanem, M. E., Marrou, H., and Sinclair, T. R. (2015). Physiological phenotyping of plants for crop improvement. Trends Plant Sci. 20, 139-144. doi: 10.1016/j.tplants.2014.11.006

Godfray, H. C., Beddington, J. R., Crute, I. R., Haddad, L., Lawrence, D., Muir, J. F., et al. (2010). Food security: the challenge of feeding 9 billion people. Science 327, 812-818. doi: 10.1126/science.1185383

Hairmansis, A., Berger, B., Tester, M., and Roy, S. J. (2014). Image-based phenotyping for non-destructive screening of different salinity tolerance traits in rice. Rice 7:16. doi: 10.1186/s12284-014-0016-3

Hannah, M. A., Wiese, D., Freund, S., Fiehn, O., Heyer, A. G., and Hincha, D. K. (2006). Natural genetic variation of freezing tolerance in Arabidopsis. Plant Physiol. 142, 98-112. doi: 10.1104/pp.106.081141

Henley, W. J. (1993). Measurement and interpretation of photosynthetic lightresponse curves in algae in the context of photoinhibition and diel changes. J. Phycol. 29, 729-739. doi: 10.1111/j.0022-3646.1993.00729.x

Humplik, J. F., Lazar, D., Husickova, A., and Spichal, L. (2015). Automated phenotyping of plant shoots using imaging methods for analysis of plant stress responses - a review. Plant Methods 11:29. doi: 10.1186/s13007-015-0072-8

James, R. A., Munns, R., Von Caemmerer, S., Trejo, C., Miller, C., and Condon, T. (2006). Photosynthetic capacity is related to the cellular and subcellular partitioning of $\mathrm{Na}, \mathrm{K}$ and $\mathrm{Cl}$ in salt-affected barley and durum wheat. Plant Cell and Environment 29, 2185-2197. doi: 10.1111/J.1365-3040.2006.01592.X

Jansen, M., Gilmer, F., Biskup, B., Nagel, K. A., Rascher, U., Fischbach, A., et al. (2009). Simultaneous phenotyping of leaf growth and chlorophyll fluorescence via GROWSCREEN FLUORO allows detection of stress tolerance in Arabidopsis thaliana and other rosette plants. Funct. Plant Biol. 36, 902-914. doi: 10.1071/Fp09095

Junker, A., Muraya, M. M., Weigelt-Fischer, K., Arana-Ceballos, F., Klukas, C., Melchinger, A. E., et al. (2015). Optimizing experimental procedures for quantitative evaluation of crop plant performance in high throughput phenotyping systems. Front. Plant Sci. 5:770. doi: 10.3389/fpls.2014.00770

Lazar, D. (2015). Parameters of photosynthetic energy partitioning. J. Plant Physiol. 175, 131-147. doi: 10.1016/j.jplph.2014.10.021 
Longenberger, P. S., Smith, C. W., Duke, S. E., and McMichael, B. L. (2009). Evaluation of chlorophyll fluorescence as a tool for the identification of drought tolerance in upland cotton. Euphytica 166, 25-33. doi: 10.1007/s10681-0089820-4

Ma, S. S., Gong, Q. Q., and Bohnert, H. J. (2006). Dissecting salt stress pathways. J. Exp. Bot. 57, 1097-1107. doi: 10.1093/Jxb/Erj098

Maxwell, K., and Johnson, G. N. (2000). Chlorophyll fluorescence - a practical guide. J. Exp. Bot. 51, 659-668. doi: 10.1093/jexbot/51.345.659

Mishra, K. B., Iannacone, R., Petrozza, A., Mishra, A., Armentano, N., La Vecchia, G., et al. (2012). Engineered drought tolerance in tomato plants is reflected in chlorophyll fluorescence emission. Plant Sci. 182, 79-86. doi: 10.1016/j.plantsci.2011.03.022

Munns, R., James, R. A., Sirault, X. R., Furbank, R. T., and Jones, H. G. (2010). New phenotyping methods for screening wheat and barley for beneficial responses to water deficit. J. Exp. Bot. 61, 3499-3507. doi: 10.1093/jxb/erq199

Munns, R., and Tester, M. (2008). Mechanisms of salinity tolerance. Annu. Rev. Plant Biol. 59, 651-681. doi: 10.1146/annurev.arplant.59.032607.092911

Muranaka, S., Shimizu, K., and Kato, M. (2002). A salt-tolerant cultivar of wheat maintains photosynthetic activity by suppressing sodium uptake. Photosynthetica 40, 509-515. doi: 10.1023/A:1024335515473

Murchie, E. H., and Lawson, T. (2013). Chlorophyll fluorescence analysis: a guide to good practice and understanding some new applications. J. Exp. Bot. 64, 3983-3998. doi: 10.1093/jxb/ert208

Oxborough, K. (2004). Imaging of chlorophyll a fluorescence: theoretical and practical aspects of an emerging technique for the monitoring of photosynthetic performance. J. Exp. Bot. 55, 1195-1205. doi: 10.1093/jxb/erh145

Oxborough, K., and Baker, N. R. (1997). Resolving chlorophyll a fluorescence images of photosynthetic efficiency into photochemical and nonphotochemical components - calculation of $\mathrm{qP}$ and Fv'/Fm' without measuring Fo'. Photosynth. Res. 54, 135-142. doi: 10.1023/A:1005936823310

Rajendran, K., Tester, M., and Roy, S. J. (2009). Quantifying the three main components of salinity tolerance in cereals. Plant Cell Environ. 32, 237-249. doi: 10.1111/J.1365-3040.2008.01916.X

Rascher, U., Liebig, M., and Luttge, U. (2000). Evaluation of instant lightresponse curves of chlorophyll fluorescence parameters obtained with a portable chlorophyll fluorometer on site in the field. Plant Cell and Environ. 23, 1397-1405. doi: 10.1046/j.1365-3040.2000.00650.x

Roy, S. J., Negrao, S., and Tester, M. (2014). Salt resistant crop plants. Curr. Opin. Biotechnol. 26, 115-124. doi: 10.1016/j.copbio.2013.12.004
Sirault, X. R. R., James, R. A., and Furbank, R. T. (2009). A new screening method for osmotic component of salinity tolerance in cereals using infrared thermography. Funct. Plant Biol. 36, 970-977. doi: 10.1071/ Fp09182

Stephan, A. B., and Schroeder, J. I. (2014). Plant salt stress status is transmitted systemically via propagating calcium waves. Proc. Natl. Acad. Sci. U.S.A. 111, 6126-6127. doi: 10.1073/Pnas.1404895111

Stepien, P., and Johnson, G. N. (2009). Contrasting responses of photosynthesis to salt stress in the glycophyte Arabidopsis and the halophyte Thellungiella: role of the plastid terminal oxidase as an alternative electron sink. Plant Physiol. 149, 1154-1165. doi: 10.1104/Pp.108.132407

Tester, M., and Langridge, P. (2010). Breeding technologies to increase crop production in a changing world. Science 327, 818-822. doi: 10.1126/ science. 1183700

Tilman, D., Balzer, C., Hill, J., and Befort, B. L. (2011). Global food demand and the sustainable intensification of agriculture. Proc. Natl. Acad. Sci. U.S.A. 108, 20260-20264. doi: 10.1073/Pnas.1116437108

Van Oosten, M. J., Sharkhuu, A., Batelli, G., Bressan, R. A., and Maggio, A. (2013). The Arabidopsis thaliana mutant air1 implicates SOS3 in the regulation of anthocyanins under salt stress. Plant Mol. Biol. 83, 405-415. doi: 10.1007/s11103-013-0099-Z

Zhang, X., Hause, R. J. Jr., and Borevitz, J. O. (2012). Natural genetic variation for growth and development revealed by high-throughput phenotyping in Arabidopsis thaliana. G3 (Bethesda) 2, 29-34. doi: 10.1534/g3.111. 001487

Conflict of Interest Statement: MTr is the owner and CEO of PSI (Photon Systems Instruments), Drasov, Czech Republic, and Dr. KP and JF are employees of his company. The other authors declare that the research was conducted in the absence of any commercial or financial relationships that could be construed as a potential conflict of interest.

Copyright (C) 2016 Awlia, Nigro, Fajkus, Schmoeckel, Negrão, Santelia, Trtílek, Tester, Julkowska and Panzarová. This is an open-access article distributed under the terms of the Creative Commons Attribution License (CC BY). The use, distribution or reproduction in other forums is permitted, provided the original author(s) or licensor are credited and that the original publication in this journal is cited, in accordance with accepted academic practice. No use, distribution or reproduction is permitted which does not comply with these terms. 